\title{
Faire connaître, éditer et exploiter les textes linguistiques français du passé : un regard rétrospectif et prospectif
}

\author{
Bernard Colombat \\ UMR 7597 «Histoire des théories linguistiques », Université Paris Diderot
}

\section{Introduction}

L'objet de cette contribution à la table ronde est de faire le point sur la connaissance que nous pouvons avoir aujourd'hui des textes linguistiques français du passé.

Nous sommes à l'heure du numérique. En fait, on ne parle plus aujourd'hui de révolution numérique, car on pense que celle-ci est largement faite. On en est moins à la constitution de corpus qu'à leur exploitation. Néanmoins, si les dictionnaires de langue française sont largement numérisés, il n'en est pas de même des grammaires, ni des remarques sur la langue (la longue tradition des « remarqueurs » inaugurée par Vaugelas), ni de ces autres outils, divers, qu'on ne sait pas trop nommer : appelés parfois « traités partiels », ce sont des traités d'épithètes, traités de synonymes, recueils de particules, etc. Tant pour les grammaires, les remarques que les outils, le corpus numérique est encore loin d'être constitué : disons qu'il est en voie de constitution.

Mais on ne saurait se contenter de cette forme de diffusion : la diffusion par l'édition classique reste incontournable, et on donnera pour preuve de cette vitalité la série de Traités sur la langue française, dans la collection des Textes de la Renaissance, publiée sous la direction de Colette Demaizière, chez Champion, depuis 1998.

Nous nous proposons d'organiser notre contribution en quatre points : comment faire connaître et décrire ces objets linguistiques ; faut-il rechercher l'exhaustivité ou la représentativité ? (nous devons approfondir le champ que recouvre ce dernier terme) ; éditer sous forme papier ; éditer sous forme numérique.

\section{Faire connaître, décrire}

Les outils existants ne manquent pas. Nous ne donnerons que quelques jalons. Certains, pour être anciens, n'ont pas perdu de leur intérêt. Pensons à la monumentale Histoire de la langue française de Ferdinand Brunot qui fait déjà une place aux théories des grammairiens du passé. Pensons aussi aux répertoires, comme celui de Stengel (1890) revu par Niederehe (1976), ou celui de Chervel $^{1}$.

Dans les années 1960, la thèse de Jean Stéfanini sur La voix pronominale en ancien et en moyen français (Gap, Ophrys, 1962) faisait une large place à l'étude des grammairiens, étude dans laquelle l'auteur s'est engagé plus avant par la suite. Mais l'ouvrage le plus emblématique est certainement la thèse de JeanClaude Chevalier, La naissance de la notion de complément, publiée chez Droz en 1968 et rééditée chez Champion en 2006. Depuis, le Que sais-je ? du même auteur, Histoire de la grammaire française, a contribué à vulgariser la connaissance véhiculée sur la tradition grammaticale française.

Aujourd'hui, la croissance de la production est exponentielle, comme le dit Chevalier dans la préface à la réédition de sa thèse :

\begin{abstract}
En 1968, je pouvais proposer une bibliographie d'ensemble qui n'était pas déraisonnable. Aujourd'hui, le champ de la discipline est devenu si vaste et si complexe, les rééditions d'ouvrages se sont tellement multipliées autant que les commentaires, qu'on peut seulement indiquer quelques voies d'accès et quelques travaux de compilation qui permettront au chercheur intéressé de s'orienter. (Chevalier 2006, p. XXII-XXIII)
\end{abstract}


Nous n'enterons évidemment pas dans une étude détaillée de ces titres. Nous ne citerons même pas de noms. Mais nous évoquerons quelques ouvrages, tout récents, qui touchent, de près ou d'un peu plus loin, au domaine : d'abord la monumentale Histoire de l'enseignement du français $d u$ XVII au XX siècle d'André Chervel (Paris, Retz, 2006), ensuite deux histoires de la langue française, la Nouvelle histoire de la langue française (Paris, Seuil, 1999) dirigée par Jacques Chaurand et les Mille ans de langue française (Paris, Perrin, 2007) d'Alain Rey, Frédéric Duval et Gilles Siouffi. Autant d'ouvrages dont le succès et la grande diffusion montrent l'intérêt d'un public large pour ce qui histoire de la langue, sinon de sa description.

Nous voudrions évoquer aussi le sort d'un répertoire, celui des 100 grammaires françaises sélectionnées pour le Corpus représentatif des grammaires et des traditions linguistiques. Rappelons le principe du projet : il s'agissait d'établir un corpus de référence pour l'étude de ce que nous appelions alors le noyau théorique du long terme, en vue d'un Dictionnaire de la terminologie linguistique (1998). La tradition française n'était pas la seule représentée, puisque nous voulions donner un aperçu global des grandes traditions linguistiques occidentales (langues anciennes, 64 titres ; langues modernes européennes, 164 titres), plus quelques traditions non occidentales - arabe, hébraïque, indienne, tamoule chinoise, japonaise - (115 titres), et quelques ouvrages relevant de la «linguistique générale », au sens large de l'expression (100 titres).

La tradition grammaticale française était donc surreprésentée: c'était voulu. Le Dictionnaire de la terminologie linguistique devait être publier en France, et la richesse de la production grammaticographique française ne pouvait être ignorée. Nous voulions donc assurer au français une place prépondérante; à titre de comparaison, nous avions demandé aux contributeurs des autres traditions européennes de sélectionner de 20 à 30 titres. La période retenue était des origines aux années 1960. Le chiffre « rond» de cent grammaires françaises (nous en donnons la liste dans l'annexe 1) n'était pas une fin en soi : c'est un peu par hasard que nous l'avons obtenu.

Comment avons-nous opéré la sélection ? de la façon la plus empirique qui soit. Nous étions aidés par le fait que nous travaillions alors à la première édition du Lexicon grammaticorum (Harro Stammerjohann dir., 1996) dont Sylvain Auroux dirigeait la partie francophone, et qu'il fallait sélectionner sinon des ouvrages, du moins des auteurs. Nous sommes arrivés à la répartition (par siècles, sans doute pas la meilleure, conceptuellement parlant, mais à tout le moins commode) suivante : jusqu'au XVI ${ }^{\mathrm{e}}$ siècle, 15 titres; XVII ${ }^{\mathrm{e}}$ siècle : 10 titres ; XVIII ${ }^{\mathrm{e}}$ siècle : 24 titres; XIX ${ }^{\mathrm{e}}$ siècle : 22 titres ; XX ${ }^{\mathrm{e}}$ siècle (jusqu'à 1960, l'une des deux limites que nous nous étions fixées, l'autre étant de ne pas avoir d'auteurs vivants) : 29 titres.

Nous avons beaucoup discuté des critères de représentativité (cf. infra), sans parvenir à une réponse homogène. Il y a des distorsions criantes. Du point de vue quantitatif par exemple, il y a une surreprésentation du XVIII ${ }^{\mathrm{e}}$ siècle par rapport au XIX ${ }^{\mathrm{e}}$. Mais elle est voulue : si le XIX ${ }^{\mathrm{e}}$ siècle est marqué par une véritable explosion des ouvrages grammaticaux (cf. les travaux d'André Chervel), ces derniers ne peuvent rivaliser, pour l'intérêt théorique, avec les "grandes » grammaires français du XVIII ${ }^{\mathrm{e}}$ siècle. Il n'empêche pas que nous avons peut-être exagérément privilégié les ouvrages pédagogiques, notamment pour le $\mathrm{XX}^{\mathrm{e}}$ siècle.

Ces notices sont parues dans le tome 1 du Corpus représentatif des grammaires et des traditions linguistiques, en 1998 (Histoire Épistémologie Langage, hors série, vol. 2). Depuis, elles sont accessibles en ligne, sur le site du Corpus de textes linguistiques fondamentaux: http://ctlf.ens-lsh.fr (rubrique 《notices $»^{2}$ ). Elles ont été partiellement mises à jour, mais une mise à niveau au point de vue bibliographique en particulier serait nécessaire. Ainsi la bibliographie associée à ces notices comporte seulement 319 références, ce qui trahit une large sous-représentation par rapport à l'ensemble des 2870 références (pour 543 notices) du Corpus représentatif des grammaires et des traditions linguistiques. Ce qui veut dire qu'il y a un travail d'actualisation important à faire, tant au niveau de la rédaction des notices qu'à celui de la mise à jour bibliographique.

Néanmoins la parution de ces notices en 1998 a eu des conséquences éditoriales; nous avons en effet été contactés par les éditions Champion, pour la création : 
- d'une base de données en ligne : au départ, cédérom, devenu DVD-rom, devenu base de données en ligne, accessible par abonnement ;

- d'une collection papier nommée Théories et descriptions de la langue française.

\section{La question de la représentativité}

Les bibliothèques se sont dites candidates à l'acquisition de la base Champion précitée uniquement si la base était exhaustive. Que veut dire « exhaustivité »? qu'on intègre l'ensemble des ouvrages concernés. Qui connaît les répertoires de Stengel (revu Niederehe) et de Chervel sait bien qu'il s'agit d'une tâche impossible. On trouvera toujours dans une bibliothèque européenne une grammaire française oubliée, sous forme de manuscrit ou d'édition rarissime, rédigée par un pédagogue méconnu. Notamment parce que les bibliothèques allemandes par exemple, ou russes (celle de Saint-Pétersbourg est particulièrement riche en éditions anciennes), cachent certainement de tels trésors.

On pourra toujours montrer l'intérêt d'un tel ouvrage retrouvé dans les archives de telle bibliothèque. Mais il nous semble que cela ne remettra pas fondamentalement en cause la vision générale qu'on peut avoir de la grammaticographie française, au moins sur le plan épistémologique ${ }^{3}$.

Ce qu'il faut chercher avant tout, c'est la représentativité, c'est-à-dire la capacité à refléter l'état des connaissance ou des techniques mise en jeu à un moment $T$. En fait les critères de représentativité pour le choix d'un ouvrage peuvent être très divers et posent des problèmes délicats.

Un ouvrage peut être représentatif :

i) d'un lieu géographique, du nombre de personnes auxquelles il s'adresse, du nombre de personnes qu'il influence réellement, d'un découpage politique, d'une volonté nationale ;

ii) d'une période donnée, et, à l'intérieur de cette période, d'un courant doctrinal, de polémiques théoriques; d'une influence durable, positive ou négative ${ }^{4}$;

iii) du but poursuivi qui peut subir d'importantes variations: commenter les textes classiques d'une langue, faire apprendre une langue particulière (comme langue maternelle ou comme langue étrangère), décrire cette langue, donner une description générale du langage ;

iv) d'une méthode ou d'une technique données, indépendamment d'une époque (formalisme, historicisme, structuralisme, etc.) ; d'une forme d'expression (traité théorique, manuel pratique, erotemata [apprentissage par questions / réponses], commentaire, etc.);

v) d'un niveau d'enseignement (élémentaire, universitaire), d'un public spécifique (les Grammaires des Dames) ;

vi) de correspondances entre deux moments donnés, quand une époque renoue avec une autre (l'humanisme avec l'Antiquité, la linguistique moderne avec les théories médiévales);

vii) d'un transfert de technologie (par ex. transfert du modèle latin dans les premiers Donat français, ou dans les premières descriptions des langues amérindiennes).

Parmi ces critères, il en est d'externes, a priori incontestables. Pourtant ils sont, comme les autres, à manier avec précaution. Prenons le nombre d'éditions. Si on suivait par exemple le principe selon lequel les ouvrages les plus importants sont ceux qui ont connu plus de x éditions, on ne retiendrait pas le Donait françois, qui n'existe que sous la forme d'un manuscrit unique, ni la grammaire de Meurier, dont il ne reste qu'un exemplaire unique... et mutilé ! Mais les grammaires de Palsgrave et de Meigret n'auraient guère plus de chance d'être retenues. Or on passerait à côté d'ouvrages incontestablement très importants.

Un autre critère pourrait être celui des références explicite à l'ouvrage chez les successeurs, par exemple le nombre de mentions (voir dans les notices du CRGTL, la rubrique « influence exercée »). Le critère se révèle pourtant imparfait dans le cadre de la transmission des textes : dans l'antiquité, au Moyen Âge ou 
encore à la Renaissance, on ne cite en principe pas ses sources et l'influence d'un auteur ne peut en aucun cas se réduire au nombre de mentions de son nom dans un ouvrage donné.

Il n'en reste pas moins que l'édition moderne d'un texte contribue, inévitablement, à le mettre en lumière, d'une manière peut-être parfois exagérée. Prenons le cas du Donait françois. Cet ouvrage est constamment cité par les historiens de la linguistique, car il présente un avantage de taille : c'est la première grammaire du français rédigée complètement en français, alors que d'autres ouvrages de la même époque sont plutôt des grammaires du latin utilisant le français comme support métalinguistique. Du coup il est promu comme « la première grammaire du français », alors que :

i) sa diffusion a sans doute été extrêmement limitée,

ii) il a été rédigé certes par « plusieurs bons clercs du langage » français, mais en Angleterre,

iii) sa composition (avec le traitement des accidents avant les parties du discours) n'est pas conforme aux canons habituels.

Il faut prendre conscience qu'éditer ou rééditer un texte, c'est en quelque sorte lui donner un coup de projecteur et, d'une façon qui n'est pas anodine, continuer à construire sa représentation.

\section{4 Éditer sous forme papier}

Certains textes grammaticaux difficiles d'accès sont devenus plus aisément accessibles : ainsi Palsgrave édité par François Génin à la fin du XIX ${ }^{\mathrm{e}}$ siècle. L'édition du Traité de la grammaire française de Louis Meigret par Franz Josef Hausmann, Tübingen, Gunter Narr, 1980, avec le texte modernisé (sauf les exemples) et une structuration des chapitres en paragraphes (alors que le texte original était en orthographe réformée et comportait de longues pages pratiquement sans alinéas) rend le texte beaucoup plus accessible aux modernes. De part le simple fait de cet accès facilité, cette édition a très certainement beaucoup contribué au regain d'intérêt des modernes pour l'écrivain lyonnais.

Signalons aussi l'édition du Liber de differentia vulgarium linguarum et Galli sermonis varietate de Charles de Bovelles, par Colette Demaizière (1972) qui a très certainement contribué à la création de la série de Traités sur la langue française, bâtie sur des principes analogues.

\subsection{Traités sur la langue française (collection des Textes de la Renaissance)}

Cette série, publiée dans la collection des Textes de la Renaissance, sous la direction de Colette Demaizière, chez Champion, réunit actuellement treize éditions de grammaires anciennes, publiées de 1998 à 2006, et largement pourvues d'index.

Une réunion « fondatrice » (mais certains étaient déjà assez avancés dans le travail) de la collection avait réuni le 16 septembre 1996 chez Colette Demaizière plusieurs éditeurs, dont: Susan Baddeley (Palsgrave), Bernard Colombat (Pillot), Geneviève Demerson (Périon), Jacques Chomarat (Henri Estienne), Colette Demaizière (qui travaillait à l'époque sur la grammaire de Sylvius, le premier volume de la série).

Les principes, pour les ouvrages en latin, les premiers de la série, étaient les suivants :

Introduction (éléments de biographie, présentation de l'oeuvre, etc.)

Texte latin reproduit en offset sur l'original. Les corrections proposées sur le texte latin seront en notes de bas de page de ce même texte.

Traduction à la suite du texte latin et faisant apparaître les repères des pages du texte latin.

Notes en bas de page de la traduction pour éclairer le texte.

Index (noms propres et thèmes). (note de C. Demaizière, non datée, antérieure à la réunion) 
Une autre note de Colette Demaizière (datée du 24 novembre 1996) précisait :

Index. Il faut en prévoir beaucoup : index nominum, locorum, index des mots étudiés à titre d'exemples (mots français, mots latins et grecs éventuellement), terminologie grammaticale (indispensable en vue de CD Rom).

Nous donnons ci-dessous la liste des treize volumes parus, par ordre de parution :

1. Dubois, Jean, Introduction à la langue française, suivie de Grammaire (1531), éd. et trad. du latin par C. Demaizière, 1998, 448 p.

2. Estienne, Henri, Hypomneses [Recommandations sur la langue française] (1582), éd. et trad. du latin par J. Chomarat, 1999, 510 p.

3. Ramus, Petrus, Grammaire [française] (1572), éd. par C. Demaizière, 2001, 167 p.

4. Cauchie, Antoine, Grammatica Gallica (1586), éd. et trad. du latin par C. Demaizière, 2001, $544 \mathrm{p}$.

5. Estienne, Robert, Traicté de la Grammaire francoise (1557), éd. par C. Demaizière, 2003, $202 \mathrm{p}$.

6. Périon, Joachim, Dialogue de l'origine du français (1555), éd. et trad. du latin par G. Demerson \& A. Jacquetin, 2003, 827 p.

7. Palsgrave, John, L'éclaircissement de la langue française (1530), éd. et trad. de l'anglais par S. Baddeley, 2003, 775 p.

8. Pillot, Jean, Institution de la langue française (1561), éd. et trad. du latin par B. Colombat, 2003, CXIX+269+365 p.

9. Bosquet, Jean (1586) Elemens ou Institutions de la langue françoise, éd. commentée par C. Demaizière, 2005, 176 p.

10. Serreius, Joannes (1623) Grammaire française, éd. et trad. du latin par A. Jacquetin, 2005, 462 p.

11. Meurier, Gabriel (1557) La grammaire françoise contenante plusieurs belles reigles propres et necessaires pour ceulx qui desirent apprendre ladicte langue, éd. commentée par C. Demaizière, 2005, 127 p.

12. Du Vivier, Gérard (1566/1568) Grammaire françoise, suivie de Briefve institution de la langue françoise expliquée en aleman, trad. et notes de B. Hébert, 2006, 185 p.

13. Garnier, Jean (1558) Institution de la langue française, introd., trad. et notes par A. Cullière, 2006, XL+103+158 p.

D'autres volumes sont encore en cours de préparation : les Devis de la langue française d'Abel Matthieu (Alberte Jacquetin), l'Introductio ad Linguam Gallicam de Daniel Cachedenier (Colette Demaizière et Alberte Jacquetin), le Donait françois (Bernard Colombat).

Sur les volumes parus, sept ont pour métalangue le latin (Dubois, H. Estienne, Cauchie, Périon, Pillot, Serreius, Garnier), un est rédigé en anglais (Palsgrave), quatre le sont en français (Ramus, Estienne, Bosquet, Meurier, Du Vivier).

Ce qui suffit à prouver que les premières grammaires française sont pour la plupart des grammaires de « FLE » (français langue étrangère), rédigées dans une langue étrangère, dont une est dominante : le latin, massivement utilisé pour l'enseignement en Allemagne au XVI ${ }^{\mathrm{e}}$ siècle. En Angleterre, c'est l'anglais (en caractères « gothiques », c'est-à-dire Fraktur) qui est utilisé pour le monumental Esclarcissement de la langue française de Palsgrave.

Par rapport à la liste des ouvrages de la même période du CRGTL, on remarquera des ajouts : Henri Estienne et Périon (mais il ne s'agit pas à proprement parler de grammaires), Meurier, Abel Matthieu (dont les Devis sont seulement en partie aussi des ouvrages grammaticaux), Du Vivier et Serreius. 
Les principes d'édition font que si le métatexte est dans une langue autre que le français (latin, anglais), l'édition comporte la reproduction en fac-similé suivie de la traduction. Si le métatexte est en français, il n'y a pas de reproduction en fac-similé, mais une édition du texte légèrement normalisée. Ainsi la ponctuation est-elle gardée, l'orthographe d'origine aussi, mais les $i / j$ et $v / u$ sont distingués, les abréviations résolues. L'accentuation est en partie normalisée. Cette normalisation, pour légère qu'elle soit, ne va pas sans poser quelques problèmes. Pour Ramus par exemple, seule l'orthographe « classique » est conservée, alors que Ramus avait bien pris soin, dans l'édition de 1572, de mettre en deux colonnes ou en deux pages en vis-à-vis les deux orthographes, l'ordinaire et la réformée, dont on sait bien qu'il la préférait, même s'il éprouvait le besoin de la doubler, du fait de l'échec de la première tentative. Pour Ramus, il aurait été, selon nous, préférable de produire un vrai dossier de fac-similés, avec, successivement l'édition de 1562 (avec la première orthographe réformée), puis celle de 1572 (avec les deux orthographes côte à côte). Plus généralement, nous préférons aujourd'hui, pour ces textes anciens, « voir » la version originale, même si cela peut poser des problèmes techniques de volume.

L'appareil périphérique est constitué par l'introduction, les notes et les index. L'introduction est relativement brève pour la plupart des traités édités au début, plus étoffée pour certains traités édités ultérieurement : Périon 2003, Palsgrave 2003, Pillot 2003, Serreius 2005, Garnier 2006 (voir tableau cidessous). Pour les notes, on retrouve sensiblement une corrélation entre le volume de l'introduction et leur volume.

Quant aux index, leur variété est relativement importante, du fait du choix laissé à l'éditeur(/traducteur). La seule idée imposée était qu'il fallait tous les index nécessaires. Nous donnons dans le tableau cidessous l'intitulé (parfois raccourci) de ces index dans les différentes éditions.

Introduction et index dans les textes de la série Traités sur la langue française

\begin{tabular}{|c|c|c|c|c|c|c|c|c|c|c|c|c|c|}
\hline & 1 & 2 & 3 & 4 & 5 & 6 & 7 & 8 & 9 & 10 & 11 & 12 & 13 \\
\hline $\begin{array}{l}\text { Taille de l'introd. } \\
\text { (en p.) }\end{array}$ & 19 & 24 & 23 & 25 & 26 & 60 & 42 & 119 & 20 & 77 & 26 & 19 & 40 \\
\hline Fac-similé & $\mathrm{x}$ & & & $\mathrm{x}$ & & $\mathrm{x}$ & $\mathrm{x}$ & $\mathrm{x}$ & & $\mathrm{x}$ & & & $\mathrm{x}$ \\
\hline INDEX & & & & & & & & & & & & & \\
\hline $\begin{array}{l}\text { Adages, locutions } \\
\text { et proverbes }\end{array}$ & & $\mathrm{x}$ & & & & & & & & & & & \\
\hline $\begin{array}{l}\text { Formes } \\
\text { graphiques } \\
\text { françaises }\end{array}$ & & & & & & & $\mathrm{x}$ & & & & & & \\
\hline $\begin{array}{l}\text { Dialectes } \\
\text { langues }\end{array}$ & & $\mathrm{x}$ & & & & & & $x^{5}$ & & & & & \\
\hline $\begin{array}{l}\text { Lieux, peuples, } \\
\text { langues }\end{array}$ & & & & & & & & & & & & & $\mathrm{x}$ \\
\hline $\begin{array}{l}\text { Mots français } \\
\text { cités en ex. }\end{array}$ & $\mathrm{x}$ & $\mathrm{x}$ & $\mathrm{x}$ & $\mathrm{x}$ & $\mathrm{x}$ & $\mathrm{x}$ & & $\mathrm{x}$ & $\mathrm{x}$ & $\mathrm{x}$ & $\mathrm{x}$ & & $\mathrm{x}$ \\
\hline Mots grecs & $\mathrm{x}$ & $x x^{6}$ & & & & $\mathrm{x}$ & & & & & & & \\
\hline $\begin{array}{l}\text { Noms de } \\
\text { lieux/Géographie }\end{array}$ & $\mathrm{x}$ & & $\mathrm{x}$ & $\mathrm{x}$ & & $\mathrm{x}$ & & & $\mathrm{x}$ & $\mathrm{x}$ & $\mathrm{x}$ & & \\
\hline $\begin{array}{l}\text { Noms propres/ de } \\
\text { personnes/Auteurs }\end{array}$ & & $x^{7}$ & & & & $\mathrm{x}$ & $\mathrm{x}$ & $x^{8}$ & & $\mathrm{x}$ & & $\mathrm{x}$ & $\mathrm{xxx}^{9}$ \\
\hline
\end{tabular}




\begin{tabular}{|l|l|l|l|l|l|l|l|l|l|l|l|l|l|}
\hline $\begin{array}{l}\text { Noms de pers. ou } \\
\text { de peuples }\end{array}$ & $\mathrm{x}$ & & $\mathrm{x}$ & $\mathrm{x}$ & $\mathrm{x}$ & & & & $\mathrm{x}$ & & $\mathrm{x}$ & & \\
\hline $\begin{array}{l}\text { Noms propres } \\
\text { dans les notes }\end{array}$ & & $\mathrm{x}$ & & & & & & & & & & & \\
\hline $\begin{array}{l}\text { « Termes esquels } \\
\text { l's s'exprime » }\end{array}$ & & & & & & & & & $\mathrm{x}$ & & & & \\
\hline Termes allemands & & & & & & & & & & $\mathrm{x}$ & & & \\
\hline Termes latins & $\mathrm{x}$ & & & & & & & $\mathrm{x}$ & & & & & \\
\hline $\begin{array}{l}\text { Terminologie } \\
\text { linguistique (fr.) }\end{array}$ & $\mathrm{x}$ & & $\mathrm{x}$ & $\mathrm{x}$ & $\mathrm{x}$ & & & $\mathrm{x}$ & $\mathrm{x}$ & & $\mathrm{x}$ & $\mathrm{x}$ & $\mathrm{x}$ \\
\hline $\begin{array}{l}\text { Thèmes } \\
\text { Vocabulaire latin } \\
\text { grammatical }\end{array}$ & & $\mathrm{x}$ & & & & $\mathrm{x}$ & & $\mathrm{x}$ & & $\mathrm{x}$ & & & \\
\hline Total des index & 6 & 8 & 4 & 4 & 3 & 5 & 3 & 6 & 5 & 5 & 4 & 2 & 6 \\
\hline
\end{tabular}

La plupart de ces index ne posent pas de problèmes particuliers. Un seul - ou plus exactement deux d'entre eux - permet de soulever un problème intéressant. Celui dit de «terminologie grammaticale », dont la note citée supra disait qu'il était « indispensable en vue de CD Rom». Dans les faits, quand l'index dit de terminologie linguistique est fait sur la traduction, il ne permet en aucun cas d'explorer le texte original qui est l'objet de la base de données numérisée. C'est le « vocabulaire latin grammatical» qui le permet, pour les textes latins, étant entendu que sous les formes répertoriées (par ex. un nom au nominatif ou un verbe à l'infinitif), il y a naturellement une très grande variété de formes possibles, du fait de la richesse flexionnelle du latin. C'est un point qui avait échappé à la directrice de collection, il est vrai à un moment où l'informatisation des textes en était à ses débuts. Il est à noter aussi que l'index de terminologie linguistique ne peut pas être le retournement exact de l'index fait sur le texte original : les termes métalinguistiques dans les deux langues ne sont pas biunivoques, d'autant que la linguistique moderne a créé bon nombre de termes, voire de concepts complètement ignorés des anciens. Il n'en reste pas moins que chacun des deux index a sa légitimité, sinon sa nécessité.

\subsection{Grammaire françaises des $\mathrm{XVII}^{\mathrm{e}}$ et $\mathrm{XVII}{ }^{\mathrm{e}}$ siècles}

Un relais à cette collection est constitué par deux séries de la collection « Théories et descriptions de la langue française »: la première, intitulée, "Grammaire françaises des XVII ${ }^{\mathrm{e}}$ et XVIII ${ }^{\mathrm{e}}$ siècles », est dirigée par B. Colombat et J.-M. Fournier; la seconde, intitulée «Remarques et observations sur la langue française », est dirigée par W. Ayres-Bennett.

Nous donnons ici, sous une forme résumée, les principes élaborés pour la série «Grammaire françaises des XVII ${ }^{\mathrm{e}}$ et XVIII ${ }^{\mathrm{e}}$ siècles » (mais la série « Remarques et observations sur la langue française » repose sur des principes similaires) et dans l'annexe 2 la liste des textes actuellement retenus.

La collection proposera l'édition critique, réalisée par des spécialistes du domaine, des textes grammaticaux français publiés au cours des $\mathrm{XVII}^{\mathrm{e}}$ et $\mathrm{XVIII}^{\mathrm{e}}$ siècles. L'objectif est de constituer une édition critique de référence de ces textes. Elle bénéficie naturellement de l'expérience acquise par le développement de la série Traités sur la langue française.

Le texte retenu sera évidemment celui de la dernière édition publiée du vivant de l'auteur. L'édition comportera un apparat critique et des notes visant à mettre en évidence les relations existant entre le texte édité et la tradition grammaticale dans laquelle il prend place. Elles devront notamment permettre d'identifier : 
- les innovations sur les plans terminologique et notionnel ;

- la position de l'auteur à propos des questions grammaticales lorsqu'elles constituent la matière d'un débat (par exemple, la règle d'emploi du passé simple et du passé composé, la place des formes surcomposées dans le système des temps, la question de l'article indéfini, du partitif, etc.) ;

-plus généralement les soubassements théoriques des points en discussion, en dégageant l'enjeu épistémologique d'une position, et en rappelant, chaque fois que nécessaire, le contexte théorique et épistémologique dans lequel se place le texte édité ;

- la source des formulations lorsque, comme il arrive souvent dans les textes de cette période, le texte comporte des emprunts sous la forme de séquences plus ou moins figées, paraphrastiques, à la tradition ;

- à l'inverse, on indiquera également les apports qui ont exercé une influence sur les grammairiens postérieurs ;

- enfin, il pourra arriver que les notes se bornent à élucider des notions.

Il faut donc prévoir un volume de notes assez consistant, qui pourra constituer un volume égal à celui du texte de la grammaire.

\section{Présentation matérielle}

Le principe d'une édition en fac-similé nous semble devoir être écarté en raison des difficultés que poserait l'insertion dans le corps du texte des appels de note, des variantes, etc. Du fait que les éditions Champion établissent une édition numérisée pour le cédérom (voir infra), elles mettront à la disposition de chaque auteur-éditeur cette version qui servira de base à l'édition critique.

Les éditions proposeront un texte reproduit à l'identique de la dernière édition du vivant de l'auteur, ou de la meilleure édition. Lorsque les textes ont connu plusieurs éditions, il faudra adopter une présentation qui rende aisée la consultation des notes et des variantes. Le principe retenu est de partir de l'édition de référence numérisée et de mettre en note :

- les variantes par rapport aux autres éditions; des annexes pouvant en outre recueillir les passages faisant l'objet des modifications les plus importantes (avec, par exemple, deux textes en regard, pour comparaison directe) ;

- tous les éclaircissements nécessaires à la compréhension du texte et à sa situation dans l'historiographie de la tradition grammaticale française ou générale. Quand un point mérite un traitement particulier, il est prévu de l'aborder dans l'introduction, ce pour éviter une annotation trop lourde, qui risquerait de devenir difficilement lisible.

Cette introduction, qui peut être d'un volume conséquent, doit donner tous les éléments nécessaires sur le plan de :

- la biographie/bibliographie de l'auteur,

- la situation de l'ouvrage dans son contexte épistémologique,

avec une attention particulière aux points suivants :

- l'élaboration des concepts linguistiques,

- l'élaboration de la terminologie grammaticale et linguistique,

- l'illustration par les faits de langue (choix et formes des exemples).

Des index nombreux sont à prévoir, notamment :

- un index des langues utilisées et leur répartition dans l'ouvrage ;

- un index des exemples et une évocation de leur histoire ; 
- un index des termes, des notions de linguistique et de logique ;

- un index des noms propres (auteurs cités dans l'ouvrage, éventuellement dans les notes).

\section{5 Éditer en ligne et exploiter électroniquement}

Internet fournit un accès aisé à bon nombre de textes linguistiques français. La plupart des grammaires françaises sont accessibles, en mode image, par le biais du serveur électronique de la BnF, Gallica (http://gallica.bnf.fr)), ou d'autres sites, comme celui des Bibliothèques Virtuelles Humanistes (http://www.bvh.univ-tours.f). Il existe d'autres sites, comme celui de Google Recherche de livres (http://books.google.fr), ou encore des sites développés par des particuliers, comme, pour la grammaire française du XIX ${ }^{\mathrm{e}}$ siècle, celui de Jacques-Philippe Saint-Gérand (http://www.chass.utoronto.ca/epc/langueXIX/)... la liste n'est pas fermée. Dans les notices descriptives du site CTLF, nous nous efforçons de mettre à jour les liens vers ces éditions.

La plupart des ces sites proposent les textes en mode image, certains avec un OCR localisé qui permet un minimum de recherches avec une fiabilité moyenne; plus rares sont les sites à proposer les oeuvres en mode texte.

Nous évoquerons ici deux projets parallèles, mais non concurrents, de numérisation et de mise en ligne de textes linguistiques français. Le premier est un projet de publication de textes de «linguistique générale ». Il comporte des textes linguistiques rédigés en français, mais aussi dans d'autres métalangues : anglais, allemand, espagnol, italien, russe. Il est le prolongement du programme Corpus de textes linguistiques fondamentaux déjà évoqué.

Le second est soutenu par les éditions Champion, il est parallèle à la collection papier déjà évoquée et il en est le complément. Portant le même titre que la collection papier, "Théories et descriptions de la langue française » (dir. B. Colombat \& J.-M. Fournier), il se subdivise actuellement en deux projets, l'un intitulé "Grammaires françaises, première tranche: XIII $-\mathrm{XVII}^{\mathrm{e}}$ siècles" (mêmes directeurs), l'autre « Remarques et observations sur la langue française » (dir. W. Ayres-Bennett).

Il nous semble intéressant de récapituler les points communs et les différences entre les deux projets, du point de vue du chercheur /préparateur, sans entrer dans le détail technique.

\section{Le projet $C T L F / D E T C O L$}

Financement par les institutions publiques, principe du libre accès aux textes sous réserve de liberté des droits

Partenaires : UMR 7597 « Histoire des théories linguistiques », Université Paris Diderot ; ENS Lettres et Sciences humaines (Lyon); ATILF (Nancy)

Durée et financement : projet en développement depuis 2002, financé sur deux supports : le projet CTLF, supporté par un CPER (contrat État Région intitulé «Accompagnement de l'arrivée de l'ENS Lettres \& Sciences Humaines dans la région Rhône Alpes », axe constitution et exploitation de corpus) (2002-2006, budget : 130000 euros) ; projet DETCOL (Développement et exploitation textuelle d'un corpus d'oeuvres linguistiques), supporté par l'ANR (2007-2010, budget global : 160000 euros)

Le portail « images»

Notice descriptive de l'ouvrage et notice technique

Constitution d'une table des matières «dépliable/repliable» (en ligne, jusqu'à 6 niveaux de profondeur)

Images associées à cette table des matières 
Est envisagée la possibilité d'un OCR localisé (du type utilisé pour le portail Persée)

Le portail « textes » (c'est-à-dire textes numérisés et balisés)

Les étapes

Océrisation des volumes

Traitement initial

Extraction des textes (opérateur)

Structuration des textes

Correction des textes

Conversion et mise en ligne des textes corrigés

Évaluation des objectifs recherchés par les chercheurs pour le balisage

Balisage opéré par Nancy

Rôle du chercheur/préparateur : il est associé, dès le départ, à la procédure technique et bénéficie d'une assistance à la structuration et à la correction, par divers outils utilisant des macros de Word, notamment une barre d'outils et une «boite à outils » lui permettant de structurer ou de corriger le texte plus rapidement.

\section{Le projet Champion}

Financement sur fonds privés, par la société Champion électronique, achat éventuel des droits, accès à la base par abonnement payant

Durée : les premiers contacts remontent à 1999 ; une première tranche de la base est prévue pour ouverture fin 2008

Principales étapes (nous neutralisons, pour simplifier, le rôle des directeurs de collection) :

1. Les Éditions Champion fournissent à chaque éditeur scientifique une copie papier des textes à éditer, dont il est gardé une copie vierge (pour archivage).

2. L'éditeur scientifique remet à Champion une copie surlignée de ce texte, selon des principes d'édition précis, accompagnée d'une notice descriptive et d'une table des matières de l'ouvrage (3 niveaux maximum).

3. Sur la base de cette version surlignée, les Éditions Champion font saisir et baliser (par deux opérateurs de saisie, en double aveugle, vérification par un $3^{\mathrm{e}}$ opérateur) le texte qui est rendu ensuite à l'éditeur scientifique sous une forme demi-débalisée.

4. L'éditeur scientifique corrige le texte (en utilisant un tirage sur papier) et vérifie le balisage, les listes d'exemples.

5. L'éditeur scientifique établit différents thesaurus : un thésaurus des auteurs ; un thesaurus des titres d'oeuvres citées ; un thesaurus de la métaterminologie de l'ouvrage.

6. L'éditeur scientifique utilise ce même texte numérisé pour établir l'édition critique de l'ouvrage sur papier dans les séries Grammaires françaises des XVII et XVIII siècles et Remarques et observations sur la langue française.

Rôle du chercheur /préparateur : il n'est pas associé à la procédure technique. Il ne travaille que sur des versions papier (texte original, puis texte demi-débalisé) S'il constate des erreurs ou des anomalies dans le balisage, il ne doit en aucun cas intervenir directement sur le texte balisé, mais fournir séparément une liste des erreurs ou anomalies. 


\section{Exploiter}

Dans le projet $C T L F$, mis à part les titres, les mises en valeur par le gras, l'italique, etc., les objectifs de balisage et d'exploitation sont encore en cours de définition. Il est prévu de pouvoir rechercher des contextes selon des procédures similaires à celles utilisés par la base Frantext.

Dans le projet Champion, les objectifs sont déjà définis :

- $\quad$ repérage des exemples, en indiquant la langue (actuellement 47 langues ou dialectes répertoriés), éventuellement l'auteur, le texte ;

- repérage des citations, en indiquant la langue, l'auteur, le texte ;

- repérage des auteurs (en distinguant auteurs sources d'exemples explicites ou adaptés, auteurs sans exemple, autorités sur la langue sources de citations explicites ou d'opinions, autorités sur la langue sans citation, autres noms de personnages cités);

- repérage des oeuvres (en distinguant titres des ouvrages sources d'exemples, titres des ouvrages sources de citations ou d'opinions, titres des ouvrages cités qui ne sont ni source d'exemple, ni source de citation);

- repérage fin du métalangage utilisé dans l'ouvrage.

Les deux programmes avancent «à leur rythme ». Ils font intervenir des chercheurs préparateurs, et, pour le projet CTLF, des chercheurs concepteurs / informaticiens, des vacataires, qui doivent recevoir une formation minimale. L'avenir nous dira si c'est le projet soutenu par l'éditeur privé ou celui sur fonds publics qui arrivera le premier à son terme, mais ce type d'entreprise prend nécessairement du temps, un temps que ne laissent nullement supposer des projets de numérisation rapide type Gallica, ou Google Recherche de livres. Face à ceux-là, il est évident que c'est dans la valeur ajoutée (par la facilité de consultation, l'enrichissement des textes, les possibilités d'interrogation multiples, etc.) que réside l'intérêt de ces programmes dévoreurs de temps et d'énergie.

\section{Annexes}

Annexe 1. Les cent grammaires françaises décrites dans le Corpus représentatif des grammaires et des traditions linguistiques, tome 1, Histoire Epistémologie Langage, Hors série $\mathbf{n}^{\circ}$ 2, 1998), p. 111-276.

\section{Ouvrages des $X I I I^{\mathrm{e}}-X V^{\mathrm{e}}$ siècles : 3 titres :}

(1) 2101. [Donat], Quantes parties d'oraison sont? (mss. et incunables des XIII ${ }^{\mathrm{e}}-\mathrm{XV}^{\mathrm{e}} \mathrm{s}$.).

(2) 2102. [anonyme], En / Par quantes manieres doit on commencer son latin? (mss. et éd. du XVI S.).

(3) 2103. [Barton, J.] (ca. 1409) Donait françois [éd. P. Swiggers, « Le Donait françois: la plus ancienne grammaire du français », 1985, Revue des langues romanes, 89, p. 235-251].

\section{Ouvrages du XVI ${ }^{\mathrm{e}}$ siècle : 12 titres :}

(4) 2201. [anonyme], Principes en françois / Principes et premiers elemens de la langue latine (entre 1510 et 1586).

(5) 2205. Palsgrave, John (1530) Lesclaircissement de la langue française, Londres, R. Pynson et J. Haukis [Slatkine Reprints, Genève, 1972].

(6) 2203. Sylvius, Jacobus Ambianus (1531) In linguam Gallicam isagoge, Paris, R. Estienne [Slatkine Reprints, Genève, 1971]. 
(7) 2204. Donat, Aelii Donati De octo partibus orationis libellus, in dialogi formam Latinam et Gallicam redactus, Paris, François / Charles Robert II Estienne (entre 1545 et 1585).

(8) 2205. Meigret, Louis (1550) Le tretté de la grammere françoeze, Paris, C. Wechel [Slatkine Reprints, Genève, 1972 ; The Scholar Press, European Linguistics, 1969].

(9) 2206. Pillot, Jean (1550) Gallicae linguae institutio, latino sermone conscripta, Paris, Stephanus Grouleau [Slatkine Reprints, Genève, 1972].

(10) 2207. Estienne, Robert (1557) Traicté de la grâmaire Francoise, Paris, R. Estienne [Slatkine Reprints, Genève, 1972].

(11) 2208. Garnier, Jean (1558) Institutio gallicae linguae in usum iuuentutis Germanicae, Genève, Jo. Crispinus [Slatkine Reprints, Genève, 1972].

(12) 2209. Ramus, Petrus (1562) Gramere, Paris, A. Wechel [Slatkine Reprints, Genève, 1972].

(13) 2210. Caucius, Antonius (1586 [1570]) Grammaticae gallicae libri tres, Strasbourg, B. Iobinus [Slatkine Reprints, Genève, 1968, avec les Hypomneses de Gallica lingua de Henri Estienne].

(14) 2211. Ramus, Petrus (1572) Grammaire, Paris, A. Wechel [Slatkine Reprints, Genève, 1972].

(15) 2212. Bosquet, Jean (1586) Elemens ou institutions de la langue françoise, Mons, Charles Michel [Slatkine Reprints, Genève, 1972].

\section{Ouvrages du XVII ${ }^{\mathrm{e}}$ siècle : 10 titres :}

(16) 2301. Maupas, Charles (1618 [1607]) Grammaire et syntaxe françoise, Orléans, O. Boynard [Slatkine Reprints, Genève, 1973].

(17) 2302. Oudin, Antoine (1640 [1632]) Grammaire françoise rapportée au langage du temps, Paris, A. de Sommaville [Slatkine Reprints, Genève, 1972].

(18) 2303. Irson, Claude (1656) Nouvelle methode pour apprendre facilement les principes et la pureté de la langue française, Paris, l'auteur. (ALF 201) [Slatkine Reprints, Genève, 1973].

(19) 2304. Arnauld \& Lancelot (1676 [1660]) Grammaire Générale et Raisonnée, Paris, P. Le Petit [Ed. par H. E. Brekle, Grammatica Universalis, 1, 1966].

(20) 2305. La Grue, Thomas de (1669) La vraye Introduction à la Langue françoise, Amsterdam, S. Imbrechts. (ALF 208).

(21) 2306. Chiflet, R. P. Laurent (1668) Essay d'une parfaite Grammaire de la langue françoise, Paris, P. Mauger [Slatkine Reprints, Genève, 1973].

(22) 2307. Vairasse d'Allais, Denis (1681) Grammaire Méthodique contenant en abrégé les Principes de cet art et les règles les plus nécessaires à la langue française, Paris, l'auteur.

(23) 2308. Mauger, Claude $\left(1684,11^{\mathrm{e}}\right.$ éd. [3 $3^{\mathrm{e}}$ éd. : 1658]) French Grammar with additions, Londres. (ALF 263).

(24) 2309. D’Aisy, Jean (1685) Le Génie de la Langue françoise, 2 vol., Paris, Laurent d'Houry [Slatkine Reprints, Genève, 1972 ; ALF 8]).

(25) 2310. Dangeau, Abbé Louis de Courcillon de (1694) Essais de grammaire, contenus en trois lettres, d'un académicien à un autre académicien, Paris, J.-B. Coignard (Opuscules sur la grammaire, rééd. par Manne Ekman, Uppsala, 1927, Almqvist et Wiksells [Slatkine Reprints, Genève, 1969]).

\section{Ouvrages du XVIII ${ }^{\mathrm{e}}$ siècle : 24 titres :}

(26) 2401. Régnier-Desmarais, Abbé François-Séraphin (1706) Traité de la Grammaire françoise, Paris, Coignard [rééd. 1973]. 
(27) 2402. Buffier, le Père Claude (1709) Grammaire françoise sur un plan nouveau pour en rendre les principes plus clairs et la pratique plus aisée, Paris, N. Le Clerc et al.

(28) 2403. Vallange, de (1721) Grammaire françoise raisonnée, qui ensègne la pureté et la délicatesse de la langue avec l'orthographe, et qui sert de clé au latin et aux autres langues, Paris, C. Jombert.

(29) 2404. Du Marsais, César Chesneau (1729-56 [1987]) Les véritables principes de la grammaire et autres textes, éd. F. Douay-Soublin, Paris, Fayard.

(30) 2405. Restaut, Pierre (1730) Principes généraux et raisonnés de la Grammaire française, Paris, J. Desaint.

(31) 2406. Val(1)art, Abbé Joseph (1744) Grammaire Françoise, Paris, Desaint et Saillant.

(32) 2407. Girard, Abbé Gabriel (1747) Les vrais Principes de la Langue françoise, 2 vol., Paris, Le Breton [éd. P. Swiggers, Droz, Genève].

(33) 2408. Sauvage de Villaire (1749) Abrégé de la grammaire françoise pour ceux qui n'ont point étudié, Paris, G. Desprez.

(34) 2409. Wailly, Noël-François de (1754) Grammaire françoise ou La manière dont les personnes polies et les bons auteurs ont coutume de parler et d'écrire, Paris, Barbou.

(35) 2410. D’Acarq, Jean-Pierre (1760) Grammaire française philosophique, 1760, Genève, Moreau \& Lambert.

(36) 2411. Beauzée, Nicolas (1767) Grammaire Générale ou exposition raisonnée des éléments nécessaires au langage pour servir de fondement à l'étude de toutes les langues, 2 vol., Paris, J. Barbou [Rééd. par B. E. Bartlett, Grammatica Universalis, 8, 1974].

(37) 2412. Demandre, A. (1769) Dictionnaire de l'élocution françoise, contenant les principes de grammaire, logique, rhétorique, versification et généralement les règles nécessaires pour écrire et parler correctement le françois, Paris, Lacombe, ALF [106.

(38) 2413. Condillac (1775) Grammaire [Tome I du Cours d'étude pour l'instruction du Prince de Parme], Parme, Imprimerie Royale [Rééd. par U. Ricken, Grammatica Universalis, nº 19, 1986].

(39) 2414. Domergue, Urbain (1782 [1778]) Grammaire française simplifiée, Paris, Durand neveu.

(40) 2415. Lhomond, Charles-François (1780) Elémens de grammaire française, Paris, Colas.

(41) 2416. Fabre, Abbé (1787) Syntaxe française, ou nouvelle grammaire simplifiée, Paris, Périsse.

(42) 2417. Blondin, Jean-Noël (1817 [1789]) Grammaire française démonstrative, $7^{\mathrm{e} e ́ d ., ~ P a r i s, ~}$ l'Auteur.

(43) 2418. Chemin-Dupontès, Jean-Baptiste (an VI [1794]) Principes de la grammaire française, $7^{\mathrm{e}}$ éd., Paris, Courrier de la Librairie.

(44) 2419. Lévizac, Jean-Pont-Victor Lacoutz, Abbé de (1822 [1797]) L'Art de parler et d'écrire correctement la langue française, ou Grammaire philosophique et littéraire de cette langue [...], 2 vol., Paris, Rémont.

(45) 2420. Caminade, Marc Alexandre, dit Caminade-Chatenay, ou le Citoyen Caminade (an VII [1798]) Premiers Elémens de la langue française, 2 vol., Paris, Agasse.

(46) 2421. Sicard, Abbé Roch-Ambroise-Cucurron (1808 [1798]) Elémens de grammaire générale appliquée à la langue française, 2 vol., Paris, Deterville.

(47) 2422. Loneux, Eugène (1799) Grammaire générale appliquée à la langue française, Liège, Bassenge \& Paris, Maradan. 
(48) 2423. Serreau, Jean-Edmé (1799) Grammaire raisonnée, ou Principes de la langue française, Paris, Richard, Caille \& Ravier.

(49) 2424. Silvestre de Sacy, A.-I. (1799) Principes de Grammaire générale mis à la portée des enfants et propres à servir d'introduction à l'étude de toutes les langues, et notamment de la langue française, Paris, Fuchs.

\section{Ouvrages du XIX ${ }^{\mathrm{e}}$ siècle : 22 titres :}

(50) 2501. Boinvilliers [pseud.], Jean-Etienne-Judith Forestier (1818 [1802]) Grammaire raisonnée, ou Cours théorique et pratique de la langue française, 2 vol., Paris, Barbou.

(51) 2502. Castille, Jean-Baptiste (1802) Grammaire françoise simplifiée, Paris, l'Auteur.

(52) 2503. Destutt de Tracy, Antoine Louis Claude (1817 [1803]) Eléments d'idéologie, seconde partie, Grammaire, Paris, Veuve Courcier [Reprod. anastatique avec notice de H. Gouhier, Paris, Vrin, 1970].

(53) 2504. Lhomond, Charles-François / Letellier Charles-Constant (an XIII [1805]) Grammaire française de Lhomond, à l'usage des lycées, nouvelle édition revue, corrigée et augmentée par Ch.C. Letellier, Paris, Le Prieur.

(54) 2505. Lemare, Pierre-Alexandre (1807) Cours théorique et pratique de la langue française [...], 2 vol. réunis en un, Paris, l'Auteur.

(55) 2506. Lhomond, Charles-François / J.-B. Chemin-Dupontès (1810) Grammaire pratique élémentaire, ou Principes de la langue française, $13^{\mathrm{e}}$ éd., chez l'auteur.

(56) 2507. Lhomond, Charles-François / Letellier Charles-Constant (1811) Grammaire française de Lhomond, à l'usage des lycées, douzième édition entièrement refaite, Paris, Le Prieur, Belin et chez l'auteur.

(57) 2508. Estarac, F. A. (1811) Grammaire générale, 2 vol., Paris, Nicolle.

(58) 2509. Girault-Duvivier, Charles-Pierre (1840 [1811]) Grammaire des grammaires ou analyse raisonnée des meilleurs traités sur la langue française, $9^{\mathrm{e}}$ éd., 2 vol., Paris, A. Cotelle.

(59) 2510. Noël, François-Joseph-Michel \& Chapsal, Charles-Pierre (1834 [1823]) Nouvelle grammaire française, Paris, Maire-Nyon, Roret.

(60) 2511. Serreau, Jean-Edme \& Boussi François-Narcisse (1824) La grammaire ramenée à ses principes naturels, ou Traité de grammaire générale appliquée à la langue française, Paris, Pélicier.

(61) 2512. Boniface, Alexandre (1836 [1829]) Abrégé de la Grammaire française méthodique et raisonnée, Paris, Delalain.

(62) 2513. Bescherelle, Louis-Nicolas, dit Bescherelle Aîné \& Bescherelle, Henri-Honoré, dit Bescherelle Jeune (1841 [1834]) Grammaire Nationale, ou Grammaire de Voltaire, de Racine, de Fénelon, ouvrage publié par MM. Bescherelle aîné, Bescherelle jeune et Litais de Gaux et CéphasSimon, Paris, L. Bourgeois-Maze.

(63) 2514. Landais, Napoléon (1835) Grammaire générale des grammaires françaises [...], Paris, Bureau Central.

(64) 2515. Vanier, Victor-Augustin (1836) Dictionnaire grammatical, critique et philosophique de la langue française, Paris, l'Auteur.

(65) 2516. Guérard, Michel (1884, $31^{\mathrm{e}}$ éd. [1852]) Cours complet de langue française, Grammaire et compléments, Paris, Delagrave. 
(66) 2517. Brachet, A. (1868, $2^{\mathrm{e}}$ éd. [1867]) Grammaire historique de la langue française, Paris, Hetzel.

(67) 2518. Larive \& Fleury [pseudonyme de Merlette, Auguste et Hauvion aîné] (1871) La première année de grammaire, Paris, Colin.

(68) 2519. Larousse, P. (1880, $20^{\mathrm{e}}$ éd. [1868]) Grammaire supérieure, Paris, Larousse.

(69) 2520. Chassang, A. (1881, $5^{\mathrm{e}}$ éd. [1880]) Nouvelle grammaire française, cours supérieur, Paris, Garnier Frères.

(70) 2521. Clédat, Léon (1894) Grammaire raisonnée de la langue française, Paris, Le Soudier.

(71) 2522. Darmesteter, Arsène (1894-1897) Cours de grammaire historique de la langue française, 4 vol., Paris, Delagrave.

\section{Ouvrages du $\mathrm{XX}^{\mathrm{e}}$ siècle (jusqu'en 1960) : 29 titres :}

(72) 2601. Nyrop, Kristoffer (1899-1930) Grammaire historique de la langue française, 6 vol., Copenhague, Gyldendal.

(73) 2602. Gilliéron, Jules \& Edmont, Edmond (1902-1912 ; 1920) Atlas linguistique de la France, 9 vol. + Supplément, Paris, Champion. ; 1920 cartes.

(74) 2603. Dussouchet, J. (1908, $2^{\mathrm{e}}$ éd. [1904]) Cours primaire de grammaire française, Cours supérieur et complémentaire, Paris, Hachette.

(75) 2604. Sudre, Léopold (1907) Cours de grammaire française; Grammaire française, cours supérieur, Paris, Ch. Delagrave.

(76) 2605. Crouzet, Paul, Berthet, G. \& Galliot M. (1922, $11^{\mathrm{e}}$ éd. [1909]) Grammaire française simple et complète pour toutes les classes (garçons et filles), Toulouse, E. Privat.

(77) 2606. Brunot, Ferdinand \& Bony, Nicolas (1908) Cours de langue française, Paris, Armand Colin.

(78) 2607. Maquet, Ch., Flot, Léon \& Roy, L. (1912) Cours de langue française, Cours moyen, Paris, Hachette.

(79) 2608. Calvet, J. \& Chomprez, C. (1917) Grammaire française, cours supérieur, Paris, de Gigord.

(80) 2609. Lanusse, M. \& Yvon, H. (1914) Cours complet de grammaire française, Paris, Librairie Classique Eugène Belin.

(81) 2610. Brunot, Ferdinand (1953 [1922]) La pensée et la langue, Paris, Masson et Cie.

(82) 2611. Radouant, René, (1922) Grammaire française, Paris, Hachette.

(83) 2612. (anonyme) (1932) Grammaire de l'Académie française, Paris, Firmin Didot.

(84) 2513. Brunot, Ferdinand (1949 [1887]) Précis de grammaire historique de la langue française, Paris, Masson [3e édition par Ferdinand Brunot \& Charles Bruneau, Paris, Masson, retirage 1969].

(85) 2614. Michaut, G. et Schricke, P. (1934) Grammaire française, Cours complet, Paris, Hatier.

(86) 2615. Gaiffe, Felix - Maille, Ernest - Breuil, Ernest - Jahan, Simone - Wagner, Léon - Marijon, Madeleine (1936) Grammaire Larousse du XXe siècle, Paris, Larousse.

(87) 2616. Grévisse, Maurice (1975 [1936]) Le bon usage, Grammaire française avec des remarques sur la langue française d'aujourd'hui, Gembloux, Duculot.

(88) 2617. Damourette, Jacques \& Pichon, Ernest (1911-1940) Des mots à la pensée, essai de grammaire de la langue française, 7 vol., Paris, d'Artrey. 
(89) 2618. Le Bidois, Georges et Robert (1935-1938) Syntaxe du français moderne, 2 vol., Paris, A. Picard.

(90) 2619. Aumeunier, E. \& Zevaco, Jean Dominique (1937) Grammaire française, enseignement du second degré, Paris, Hachette.

(91) 2620. Bloch, Oscar \& Georgin, René (1937) Grammaire française, Paris, Hachette.

(92) 2621. Gougenheim, Georges (1938) Système grammatical de la langue française, Paris, d'Artrey.

(93) 2622. Bruneau, Charles \& Heulluy, Marcel (1937) Grammaire française et exercices, classe de 4e $A$ et $B$, Paris, Delagrave.

(94) 2623. Dauzat, Albert (1947) Grammaire raisonnée de la langue française, Lyon, IAC (coll. Les langues du monde, vol. 1).

(95) 2624. De Boer, Cornelius (1947) Syntaxe du français moderne, Leyde, Presses Universitaires.

(96) 2625. Cayrou, Gaston - Laurent, Pierre - Lods, Jeanne (1948) Grammaire française à l'usage des classes de $4^{e}, 3^{e}, 2^{e}$ et $1^{\text {ère }}$ suivie des éléments de versification, Paris, Armand Colin.

(97) 2626. Cohen, Marcel (1948) Grammaire française en quelques pages, Strasbourg, Istra.

(98) 2627. Galichet, Georges (1950) Essai de grammaire psychologique du français moderne, Paris, P.U.F.

(99) 2628. Togeby, Knund (1965, $2^{\mathrm{e}}$ éd. [1951]) Structure immanente de la langue française, Paris, Larousse.

(100) 2629. Souché, Aimé (1964) La grammaire nouvelle et le français, Cours complet, Paris, Nathan.

Annexe 2. Collection (papier) «Théories et descriptions de la langue française », série " Grammaires françaises des $\mathrm{XVII}^{\mathrm{e}}$ et XVIII ${ }^{\mathrm{e}}$ siècles » (dir. B. Colombat et J.-M. Fournier). Liste des textes retenus.

(1) MASSET, Jean (1606) Exact et tres-facile acheminement à la langue françoise, par Jean Masset, mis en latin par le mesme autheur pour le soulagement des étrangers, Paris, David Douceur (grammaire intégrée au Thresor de la langue françoyse de Jean Nicot). Édition préparée par Serge Vlassov (Saint-Pétersbourg).

(2) MAUPAS, Charles (1618 [1607]) Grammaire et syntaxe françoise, Orléans, O. Boynard [Slatkine Reprints, Genève, 1973]. Édition en préparation par Nathalie Fournier.

(3) OUDIN, Antoine (1640) Grammaire françoise rapportée au langage du temps, Paris, A. de Sommaville [Slatkine Reprints, Genève, 1972]. Préparatrice : Bérangère Bouard.

(4) IRSON, Claude (1656 \& 1662) Nouvelle methode pour apprendre facilement les principes et la pureté de la langue française, Paris, l'auteur [Slatkine Reprints, Genève, 1973]. Édition en préparation par Simone Delesalle et Francine Mazière.

(5) ARnauld, Antoine \& LAnCElot, Claude (1676 [1660]) Grammaire Générale et Raisonnée, Paris, P. Le Petit [Éd. en fac-similé par H. E. Brekle, Grammatica Universalis, 1, 1966]. Édition en préparation par Sylvain Auroux, Bernard Colombat, Jean-Marie Fournier.

(6) ChIflet, R. P. Laurent (1668) Essay d'une parfaite Grammaire de la langue françoise, Paris, P. Mauger [Slatkine Reprints, Genève, $1973: 1^{\text {re }}$ éd. 1659]. Édition en préparation par Chantal Wionet (Univ. d'Avignon).

(7) VAIRASSE D’Allais, Denis (1681) Grammaire Méthodique contenant en abrégé les Principes de cet art et les règles les plus nécessaires à la langue française, Paris, l'auteur. Édition en préparation par Florence Lefeuvre (Univ. de Paris 3). 
(8) MAugER, Claude (1684, $11^{\mathrm{e}}$ éd. [3 $3^{\mathrm{e}}$ éd. : 1658]) French Grammar with additions, Londres, Thomas Harrison (ALF 263). Édition en préparation par Valérie Raby (Université de Reims).

(9) LA TOUCHE, Pierre de $\left(1730^{4}\right.$ [1696]) L'Art de bien parler françois, qui comprend tout ce qui regarde la grammaire..., $4^{\mathrm{e}}$ édition, Amsterdam, chez les Westeins \& Smith. Édition en préparation par Odile Leclercq (Université de Provence).

1 Chervel, André (2000 [1982], Les Grammaires françaises, 1800-1914. Répertoire chronologique, Avant-propos d'Alain Choppin, Paris, INRP, Service d'histoire de l'éducation [1 $1^{\text {re }}$ éd., 1982, 223 p. ; $2^{\mathrm{e}}$ éd., revue et augmentée, 222 p.].

2 Les rubriques et sous-rubriques des notices sous la forme papier étaient les suivantes : Biographie de l'auteur ; Biographie de l'adaptateur ; Titre ; Type de l'ouvrage ; Date de composition ou de première édition (= Original); Références de l'édition utilisée; Reproduction moderne; Diffusion; Langue(s) cibles(s) et métalangue; Sommaire ; Objectif de l'auteur ; Intérêt général ; Intérêts spécifiques (dont : Traitement des parties du discours ; Innovations terminologiques; Corpus illustratif; Indications complémentaires); Influence subie ; Influence exercée ; Renvois bibliographiques ; Auteur de la notice. Les champs ont été redéfinis pour l'édition en ligne, afin de faciliter l'exploitation numérique.

3 Et il est même indispensable de le faire : voir par ex. la mise à jour de la grammaire d'Osvald Stocker in Christelle Godat (2004) «La variété socio-régionale de français recommandée par Osvald Stocker dans sa grammaire (1549) », in Jean-Claude Arnould et Gérard Milhe Poutingon éd., Les normes du dire au XVI siècle, Paris, Champion, p. 291-303.

4 Nous entendons par les termes “ influence négative durable " le fait qu'un ouvrage soit jugé inadapté, voire néfaste, par les générations suivantes, mais que son influence reste telle que ces dernières estiment encore nécessaire d'en réfuter constamment le contenu (ainsi le Doctrinale d'Alexandre de Villedieu, considéré comme repoussoir pour les grammairiens humanistes).

5 Dialectes et langues, peuples, villes et régions.

6 Index grec double : 1 . mots grecs ; 2. citations, locutions et proverbe grecs.

7 Auteurs et oeuvres nommés par Henri Estienne.

8 Double : I. Auteurs et personnages anciens ; 2. Auteurs modernes.

9 L'index des noms de personne est triple : 1. auteurs anciens; personnages antiques et bibliques ; 2. auteurs et personnages du Moyen Âge et de la Renaissance ; 3. auteurs modernes.

10 «Mots latins cités comme exemples d'évolution phonétique ou morphologique en latin ».

11 « Termes latins traduits en français par Pillot». 